\title{
Entrevista a la Doctora Elsa Meinardi
}

Entrevistador: Édgar Orlay Valbuena Ussa (Profesor Departamento de Biología de la Universidad Pedagógica Nacional (Colombia) y Director de la revista)

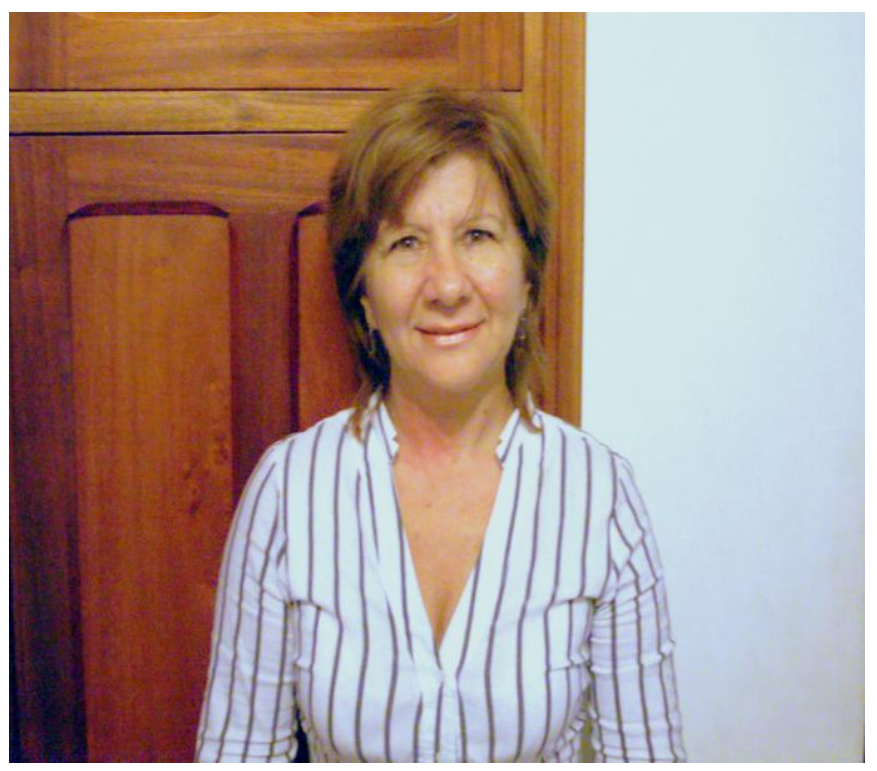

Aprovechando participación de la doctora Meinardi, el pasado mes de Octubre, en el IV Congreso Internacional de Formación de Profesores de Ciencias en Bogotá, Bio-Grafía tuvo la oportunidad de entrevistarla, abordando asuntos relativos a la Enseñanza de la Biología y más concretamente lo que atañe a la formación de profesores de Biología.

La profesora Elsa es Licenciada en Ciencias Biológicas de la Universidad de Buenos Aires y doctora en Ciencias Biológicas de la misma universidad. Actualmente coordina el grupo de investigación Didáctica de la Biología de la Facultad de Ciencias Exactas y Naturales de la Universidad de Buenos Aires.

Édgar Valbuena: ¿En qué ha consistido la experiencia de tu grupo de investigación en el trabajo que vienen realizando en centros educativos de estratos socioeconómicos de bajos de Buenos Aires?

Elsa Meinari: Podríamos hablar de las experiencias que venimos realizando, porque son varias. Intentaré resumir algunas, aunque como bien sabemos lo que ocurre en su interior es, en gran medida, intransferible.

El marco para el trabajo que venimos realizando podría resumirse bajo el título que proponemos como una pregunta: Qué formación docente para una educación científica de calidad para todos.

¿Por qué a modo de pregunta? Porque hay varios puntos incluídos en ella. Por un lado, si es deseable una formación docente de calidad para todos; por otro, si asumimos que sí, cuál es la formación docente adecuada. Podemos preguntarnos a qué educación científica debemos apuntar, qué contenidos, para qué, cómo.

Y por supuesto sin pasar por alto cuestiones más generales aún, como por ejemplo para qué sociedad. ¿Para una sociedad que persigue una escuela reproductora, que regula y se legitima con la presencia de excluídos?

$\mathrm{Y}$ en todo este conjunto de preguntas que nos hacemos y de cuyas respuestas intentamos tomar posición para poder pensar qué formación docente, nos 
preocupa que la escuela y sus docentes no contribuyan a la construcción de una institución expulsora de la calidad. Intentamos que las formas de inclusión incluyan también la calidad de los aprendizajes, que las formas de exclusión no se amparen bajo nuevos aspectos, que adquieran otras fisonomías y se sigan manteniendo las reglas normativas actuales que nos dicen quiénes merecen aprender ciencia de calidad y quiénes no.

Por eso, desde el espacio privilegiado que tenemos de formar docentes en Biología, en la Universidad de Buenos Aires, en Argentina, la Universidad pública y gratuita más grande del país, con presupuestos para la investigación, desde el grupo de trabajo que conformamos 5 docentes (cuatro de los cuales tenemos dedicaciones exclusivas para la docencia y la investigación en Didáctica de las Ciencias), pensamos que una manera útil de encausar los recursos de la sociedad es formar docentes que puedan (y si es posible quieran) trabajar en las escuelas donde más se los necesita. Allí donde asisten chicos y chicas que viven en la calle, con causas judiciales y libertad asistida, madres y padres adolescentes, agredidos -y agresivos-, que no tienen la vivencia de escuchar y ser escuchados, niños y niñas que, quizá, tengan una única oportunidad en su biografía escolar de recibir una educación científica de calidad.

Pero todo esto no puede ser encarado con un único proyecto y sin recursos, por eso en los últimos años hemos dedicado mucho esfuerzo a encausar nuestros proyectos mediante diferentes programas que organiza el Ministerio de Educación. Voy a relatar algunos.

Desde hace 3 años participamos de un programa denominado Aprender Enseñando, el cual financia con una pequeñísima suma a alumnos de profesorado para hacer tutorías en las escuelas. En nuestro caso, las tutorías se realizan en estas escuelas. Los estudiantes de profesorado cuentan con el apoyo de nuestro equipo docente, de un docente referente en las escuelas y colaborar dando asistencia dentro y fuera del aula a los/as docentes y alumnos/as según se lo requiera, ya sea para colaborar en alguna parte del trabajo dentro de los cursos o dando apoyo para los exámenes y consultas en la escuela. Los chicos saben cuándo pueden ir a consultar, hay horarios determinados para eso, o bien los docentes cuentan con apoyo para trabajar de a dos en el aula. El acercamiento que esto da a nuestros alumnos del profesorado a las necesidades y formas de trabajar en estos contextos es inenarrable aquí. Algunas experiencias pueden verse en parte relatadas en algunas publicaciones; por ejemplo en el Congreso que estamos hoy participando en Bogotá (IV Congreso Formación Docente, 2009) y publicado en la Revista TED, se presenta el relato de dos tutoras que participan desde hace dos años, en el cual cuentan cómo la experiencia de tutorías retroalimentó sus procesos de formación docente. Creo que vale la pena leerlo porque resume muchas de cosas buenas del programa.

Al mismo tiempo, tenemos en marcha en el grupo tres tesis de doctorado, una específicamente en educación sexual, en la cual estamos investigando las representaciones sobre sexualidad que integrarían el currículo oculto de docentes, en este caso de esas escuelas. Para eso, la tesista está hace tres años asistiendo y colaborando con una de las escuelas, en la cual al mismo tiempo ha hecho varias experiencias de talleres de educación sexual. Pero, no podemos encarar la educación sexual si primero, los formadores de formadores no aprendemos sobre el tema. Por eso, hemos encarado esta tesis con el propósito de estudiar nosotras el tema para luego ver cómo prescribir al respecto. Un 
punto interesante $-\mathrm{y}$ estresante- es que debemos revisar primero nuestras propias concepciones sobre sexualidad, por eso digo "personalmente movilizador".

Sumado a esto, todas las experiencias que se transitan normalmente en cualquier proceso de formación del profesorado, como observaciones de clases, planificaciones de unidades didácticas y prácticas de residencias docentes, las orientamos en estas escuelas. Nuestros alumnos y alumnas del profesorado cuentan con una lista de horarios de docentes a los que pueden contactar para ir a hacer observaciones de clases, luego pautar los temas que van a planificar y finalmente las fechas de sus prácticas docentes. Estos procesos involucran al profesorado en actividad en las escuelas, el cual se ve involucrado en un proceso de formación profesional continua.

El último trabajo que hacen los/las futuros/as profesores/as es una indagación sobre algún aspecto de la realidad escolar. Para eso, deben plantearse algún problema de indagación, los instrumentos para el relevamiento de datos, las variables relevantes del problema, las categorías y los indicadores apropiados, la bibliografía que sustenta todo el trabajo, en fin, lo que da lugar a un trabajo de indagación (una investigación limitada) sobre alguna problemática del contexto en el cual se viene desarrollando la intervención en la escuela. Esto los lleva a poner en correspondencia los marcos teóricos que han leído durante su trayecto de formación con la realidad. Aquí uno puede reconocer a autores como Perrenoud, que hablan de repensar la articulación entre teoría y práctica, o la revalorización de la escuela como ámbito de apropiación de saberes para la formación profesional docente. También podríamos hablar de un Conocimiento Didáctico del Contenido en contexto. Ponemos en acto esta idea de que la formación que se brinda al docente debe centrarse en el contexto de su desempeño.

Otra experiencia que quiero comentar y que resulta un ejemplo del trabajo que venimos haciendo consiste en la implementación de una materia que forma parte del currículo del último año, "Ambiente y salud". En la planificación pusimos en juego una concepción que desarrollamos respecto de aunar los marcos epistemológicos de ambos campos. La Didáctica de la Educación Ambiental y de la Educación para la Salud tienen -a mi criteriopoco desarrollo teórico. Se habla superficialmente de contenidos transversales, interdisciplina, y otros que no comparto (tema que no voy a desarrollar aquí), por lo cual hay una tensión entre que el docente haga interdisciplina unipersonal en el aula o caiga en un reduccionismo biologicista cuando tiene que encarar la educación en temas que, necesariamente, involucran aspectos sociales, jurídicos, éticos, culturales, además de los biológicos. Al llevar adelante el desarrollo de esta materia anual trabajamos en el relevamiento de problemas ambientales "reales" de los niños y niñas. Desde hace ya 3 años se hace este proyecto con excelentes resultados.

É.V.: Y en esa experiencia educativa tan interesante, ¿cómo se definen los contenidos de enseñanza?

E.M.: Esta es una pregunta que nos hacen muchas veces. Los contenidos que se definen vienen, en parte, decididos por el currículo oficial. La otra parte es la que se define a partir de los modelos teóricos de formación docente que adoptamos. Y esto sería general para todos los contextos. Lo que voy a mencionar no es nuevo para ustedes. ¿Hay que dar fotosíntesis? Sí, por supuesto. No vamos a hacer asistencialismo, no vamos a enseñar a 
lavarse los dientes. Vamos a hacer educación científica de la mejor calidad que podamos. Pero... ¿cuánto de fotosíntesis? ¿Lo mismo que para todos? Sí, lo mismo para niños ricos que para niños pobres ¿pero cuánto? Bueno, esto viene marcado por la necesidad de priorizar contenidos que favorezcan la comprensión. Con la contemplación de las ideas previas de los niños y los obstáculos que subyacen en ellas, la resolución de problemas, el énfasis en la comunicación, o sea, todo lo que sabemos de una educación innovadora que genere más y mejores oportunidades de participación $\mathrm{y}$, por ende, de educación. Trabajamos sobre el modelo bajo el cual saber ciencias implica: saber, hacer y comunicar. Pero aquí quiero hacer una salvedad: si en algún momento nos encontramos con que pensamos que tal vez un contenido no "sirve" para dar cuenta de algún problema, entonces vamos por todo, o sea, nos preguntamos si le servirá a alguien, en la escuela secundaria -o media- saber eso. ¿Qué problema explica? ¿De qué problema del mundo me permite dar cuenta? ¿a la comprensión de qué aporta? La conclusión es que si le sirve a estos chicos no le sirve a ningún chico o chica. Pensar los contenidos pertinentes para ellos nos hace pensar en los contenidos pertinentes para todos y todas.

E.V.: ¿En este proyecto qué se persigue respecto a la formación de profesores de Ciencias y de Biología?

Confiamos en una formación del profesorado en ciencias que apunte a una revalorización de las funciones de la escuela, tanto por los docentes como por los propios educandos, reconociendo el papel central que desempeña en la adquisición de una cultura científica. El objetivo de esta formación docente deberá centrarse en la producción y puesta en el aula de estrategias innovadoras de enseñanza de las ciencias, cuyo fin primordial sea mejorar los aprendizajes de los/las estudiantes de manera de incidir sobre la valoración de sus propios saberes, haciéndolos operativos. Se trata de lograr, mediante la formación del profesorado, la constitución de jóvenes reflexivos, críticos, autónomos, conocedores de sus derechos y capaces de demandar por los deberes de la sociedad.

Para eso, se requiere de un profesorado también reflexivo, crítico y autónomo; profesionales que puedan revisar su práctica y producir cambios. Una forma de apoyar estos procesos es constituyendo grupos de trabajo colaborativo en los cuales la articulación de la investigación didáctica con la formación inicial de los profesores y con la formación continua de los docentes en servicio, transforme los problemas del aula en problemas de investigación.

E.V. :En lo presentado en tu conferencia (en el marco del IV Congreso Internacional de Formación de Profesores de Ciencias) planteas que un referente importante en este proyecto es el del aprendizaje desde la perspectiva del cambio representacional, al respecto: ¿a qué te refieres con cambio representacional?, ¿por qué no trabajar desde la perspectiva del cambio conceptual?, ¿por qué este abordaje teórico?

E.M.: La perspectiva que nos interesa considerar es bastante reciente; propone que el cambio que se produce en el camino que va desde las ideas previas a las ideas científicas es mucho más complejo que un mero cambio de conceptos; se trataría de un verdadero cambio representacional. Los modelos que sostienen esta postura se denominan "calientes" en contraposición a los "modelos fríos" o racionales del cambio conceptual. 
Para algunos autores el cambio conceptual supone cambios en el modelo mental de una persona a medida que adquiere conocimientos. Las suposiciones ontológicas y epistemológicas contribuyen en restringir los tipos de creencias e inferencias que pueden existir en el modelo mental de una persona. Los estudiantes realizan observaciones de los fenómenos sobre la base de su experiencia en un contexto cultural. Esta experiencia origina inferencias, creencias y un conocimiento conceptual sobre los fenómenos y se ve restringida por las suposiciones epistemológicas y ontológicas (creencias básicas, categorías) de los individuos.

Otros investigadores sugieren extender el modelo de cambio conceptual, para lo cual proponen que las creencias motivacionales sobre uno y sobre el aprendizaje pueden actuar como suposiciones que dificulten o favorezcan el cambio. Y por lo tanto, proponen incluir factores cognitivos, motivacionales y sociales/contextuales al considerar el cambio que sufre -o debería sufrir- una persona en el camino que recorre -o debería recorrer- al pasar de un conocimiento ingenuo del mundo a un conocimiento científico. Es decir, involucrarse con el aprendizaje puede llevar a un cambio más profundo que el meramente conceptual.

Nuestra postura coincide con Fabre cuando dice que toda transformación de las representaciones (¿qué hay que pensar?) es igualmente una remodelación de las identidades (¿cuánto valgo?) y una puesta en cuestión de las ideas recibidas (¿qué hay que creer?). El conocimiento está vinculado con la historia de cada persona. De esta forma, las ideas del sentido común y las postinstruccionales no tendrían la misma relevancia desde el punto de vista didáctico.

\section{E.V.: En ese sentido, ¿cómo asumen el Conocimiento Escolar?}

E.M.: Proponemos que el Conocimiento Escolar es de naturaleza completamente distinta de una "idea intuitiva". El conocimiento científico escolar es, en el mejor de los casos, una transposición del científico. Probablemente el estudiante no posea modelos del mundo similares a los científicos sino, más bien, algo mucho más simple y quizá menos coherente.

Por el contrario, el conocimiento "cotidiano" es de carácter intuitivo y ha sido compartido con sus pares y con su familia (es intersubjetivo), se ha validado con la experiencia y se ha adquirido en un contexto en el cual le permitió a la persona formar parte de un grupo, ser aceptado en él. En algunos casos la impronta afectiva de estos saberes puede ser muy grande.

Cuando la enseñanza formal impone al estudiante dejar de confiar en estos referentes afectivos y socioculturales que le han permitido construir y manejar la realidad por sucesivas aproximaciones, para darle un lugar a la información que trae el maestro o la profesora, el estudiantado no logra transformar sus representaciones probablemente, entre otras cosas, porque no comprende por qué debe hacerlo.

A pesar de la importancia que estos aspectos afectivos tienen para el aprendizaje, recién hace pocos años se formulan modelos que los consideran como variables. La inclusión de la afectividad y la subjetividad entre las variables a ser investigadas en el proceso 
didáctico es probablemente un campo que veamos desarrollarse en los próximos años.

Por lo tanto, parece obvio, pero poco tenido en cuenta en las investigaciones, y aún en la práctica educativa, que debería haber alguna distinción acerca de si las ideas previas son ideas cotidianas, o si se han constituido en el marco escolar, como producto de la instrucción, o bien resultan de la interacción de ambos saberes, en el mejor de los casos.

A los fines de la educación, pensamos que no puede ser lo mismo un modelo explicativo del mundo validado socio-afectivamente, que un saber aprendido en la escuela. Si podemos diferenciarlos, nuestro proceder sobre ellos también podrá ser diferente. Más allá de que algunos los consideren "obstáculos epistemológicos", "enemigos a combatir" o "conocimientos contextualmente erróneos".

E.V.: Elsa, muchas gracias por tu tiempo, por compartir parte de los desarrollos del Grupo de Investigación que coordinas. Esperamos tenerte pronto de vuelta en Colombia. 